\title{
New Technical Solutions in the Production Method of High- Aperture Ruled Diffraction Gratings
}

\author{
Andrei Melnikov", and Anatoly Lukin \\ JSC "Scientific and Production Association "State Institute of Applied Optics", Civilian Production Department, 2, N. Lipatova st., \\ Kazan, Russia, 420075
}

\begin{abstract}
This paper presents a new approach in the production method of high-aperture diffraction gratings with high diffraction efficiency on convex and concave working surfaces with large deflection based on the use of an original pendulum-type ruling engine.
\end{abstract}

\section{Relevance}

A major optical element of a wide range of spectral equipment is a nonplanar diffraction grating with high diffraction efficiency and high aperture which, besides its primary function as a dispersive element, is involved in correcting aberrations and imaging the objects under study.

The use of these diffraction gratings is especially relevant for developing compact high-aperture spectrophotometric devices for remote probing of the Earth from the outer space, ecological monitoring, etc. Moreover, it opens up the possibility to minimize the number of optical elements in the layout of such equipment.

Two main techniques are used today to make highaperture gratings: ruling method (based on the classic Rowland ruling engines and precision lathes with numerical program control) and holographic method [16]. However, these techniques do not allow making diffraction structures on nonplanar working surfaces with large deflection ( $5 \mathrm{~mm}$ and more) while keeping a high concentration of energy in the diffraction working order and a moderate level of scattered light [7-10].

\section{A pendulum-type ruling engine}

A new technical solution suggested by us enables to overcome the aforementioned problem in the production method of high-aperture ruled diffraction gratings [11]. Figure 1 below represents a general view of a pendulumtype ruling engine for making grooved structures of ruled diffraction gratings on convex spherical or aspherical (including toroidal) working surfaces.

The blank carriage is set on a base turnable on rotation bearings in a plane perpendicular to the plane of angular auto-oscillations of the diamond carriage; the geometrical pivot axis of the blank carriage intersects the geometrical axis of angular auto-oscillations of the diamond carriage. The substrate of the manufactured convex diffraction grating is attached to the blank carriage; a diamond cutter with a hoisting and lowering mechanism is attached to the diamond carriage. The blank carriage drive is made with the function of electronic control of its turning process.

The diamond carriage is a physical pendulum, the angular auto-oscillation axis of which is secured on two rotation bearings that ensure large angular autooscillation amplitude in the swinging plane. This carriage is made as a vertical frame and equipped with a telescopic mechanism for height adjustment. Due to having a counterweight, the diamond carriage possesses the dynamic properties of a physical pendulum with a center of mass situated below the geometrical axis of its angular auto-oscillations. The movement trajectory of the diamond cutter during its cutting pass is almost equal to the curvature radius of the convex working surface of the substrate. The diamond carriage drive is intended to provide lateral motion, i.e. diamond cutter feed in the form of continuous angular oscillations, which is achieved in the auto-oscillation mode.

Unlike other known analogues, the suggested construction principles of this ruling engine enable to form grooved structures on nonplanar working surfaces almost with no deflection limitations.

The version of ruling engine construction shown in Figure 1 enables to produce diffraction gratings only on convex surfaces. To get high-aperture diffraction gratings on concave working surfaces, it is possible to use the technique of precision replication with the use of low-shrinkage polymeric compositions [12]. In this case, a convex original grating made with the suggested ruling engine can be used as a master matrix.

This two-stage approach to grating production ensures a rather high optical quality and high diffraction efficiency, as well as low cost and high productivity, which is especially important in the context of largescale production of spectral equipment of various applications.

Corresponding author: mr.melnikov@bk.ru 


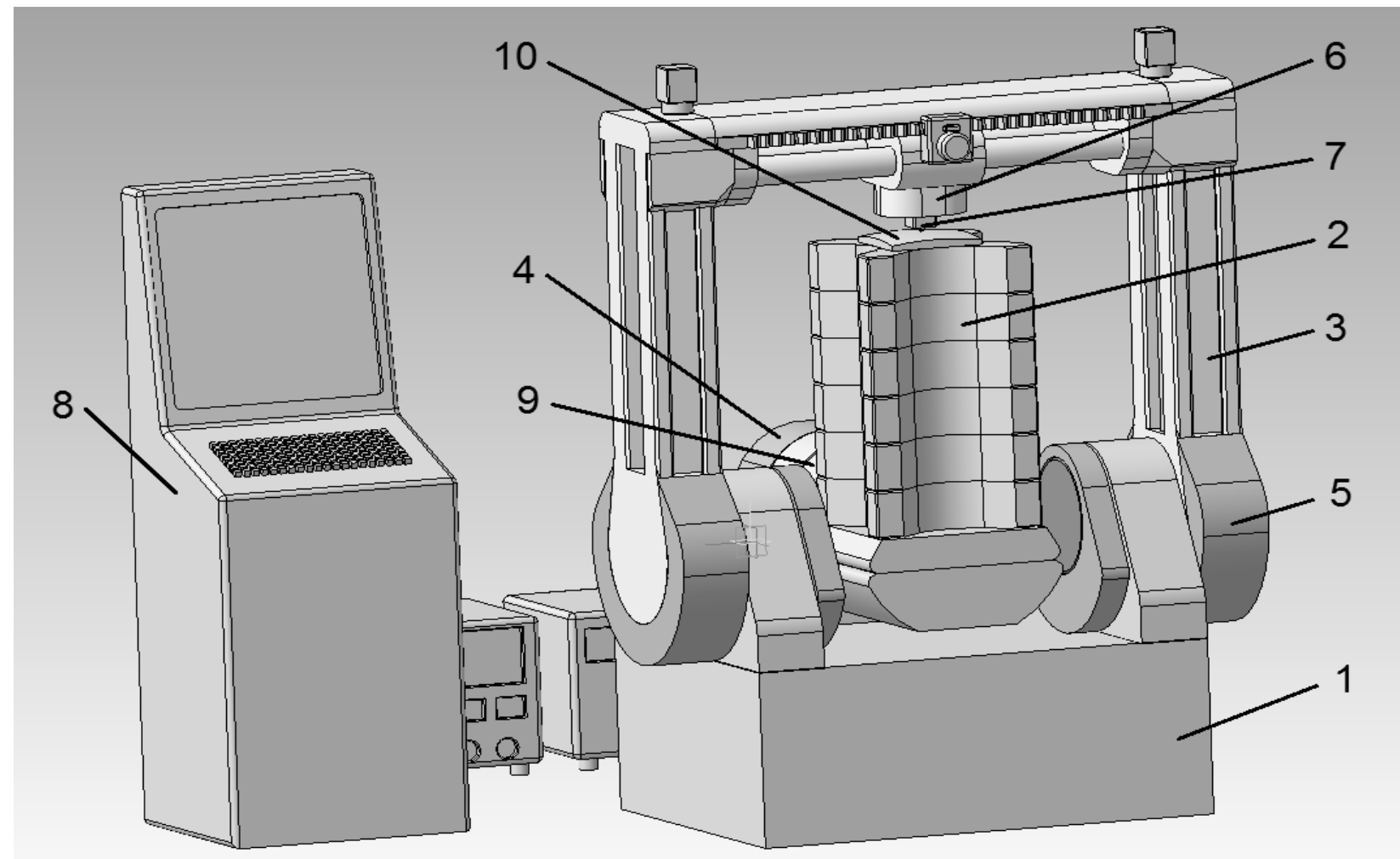

Fig. 1. The main units and elements of a pendulum-type ruling engine: 1 - base; 2 - blank carriage; 3 - diamond carriage; 4 -blank carriage drive; 5 - diamond carriage drive; 6 - diamond cutter hoisting and lowering mechanism; 7 - diamond cutter; 8 - blank carriage drive, diamond carriage drive, and diamond cutter hoisting/lowering mechanism control unit; 9 - angular position sensor of the blank carriage; 10 - substrate of the ruled diffraction grating that is being manufactured.

\section{Conclusion}

In view of this, the new suggested technical solutions open the potential to produce high-aperture diffraction gratings with high diffraction efficiency on convex and concave working surfaces for compact spectral equipment of various applications.

\section{References}

1. M.C. Hutley, Diffraction gratings (Academic Press, London, 1982)

2. F.M. Gerasimov, E.A. Yakovlev, Modern trends in the spectroscopy technique (Nauka, Novosibirsk, 1982) [in Russian]

3. T. Harada, T. Kita, Appl. Opt. 19, 3987-3993 (1980)

4. C. Palmer, Diffraction grating handbook (Newport Corporation, N.Y., 2014)

5. D. $\mathrm{Xu}$, J.D. Owen, J.C. Papa, J. Reimers, T.J. Suleski, J.R. Troutman, M.A. Davies, K.P. Thompson, J.P. Rolland, Opt. Exp. 25, 15252-15267 (2017)
6. T. Sukegawa, Yu. Okura, T. Nakayasu, Proc. SPIE 10706, 107063L-1-107063L-6 (2018)

7. JSC Shvabe Holding / JSC Scientific and Production Association "State Institute of Applied Optics" / Optic component base catalogue / URL: http://shvabe. com

8. Hitachi Group Corporation / URL: http://www.hitachi-hightech.com

9. HORIBA Jobin Yvon Ltd. Corporation / URL: http://www.horiba.com

10. Carl Zeiss Microscopy GmbH Company / URL: http://www.zeiss.de/gratings

11. A.V. Lukin, A.N. Melnikov, Useful model patent RU № 185039 dated 19 Nov 2018 [in Russian]

12. M.M. Akhmetov, A.F. Belozyorov, V.A. Baloyev, A.A. Belokopytov, I.S. Gainutdinov, V.P. Ivanov, A.V. Lukin, A.N. Melnikov, I.A. Mogilyuk, Kontenant 15, 39-42 (2016) [in Russian] 